Research Paper

\title{
Cisplatin based induction chemotherapy modified by ERCCl improved the outcome of young adults with locally advanced oral squamous cell carcinoma
}

\author{
Yin Gong $2^{2^{*}}$, Houyu Ju1 ${ }^{*}$, Guoxin Ren ${ }^{\bowtie}$, Yunteng $\mathrm{Wu}^{1}$ \\ 1. Dept. of Oral \& Maxillofacial -head and neck Oncology, Ninth People's Hospital, College of Stomatology, Shanghai Jiao Tong University School of \\ Medicine; National Clinical Research Center for Oral Diseases; Shanghai Key Laboratory of Stomatology \& Shanghai Research Institute of Stomatology, \\ Shanghai 200011, China. \\ 2. Dept. of of stomatology, First Affiliated Hospital of Soochow University, Suzhou 215000, China. \\ *Drs Gong and Ju contributed equally to this work. \\ $\triangle$ Corresponding author: Wu yun-teng: wuyunteng731@163.com or Ren guo-xin: renguoxincn@hotmail.com. 639 Zhi Zao Ju Road, Shanghai, PR China. Tel.: \\ +862150398070 . \\ (c) Ivyspring International Publisher. This is an open access article distributed under the terms of the Creative Commons Attribution (CC BY-NC) license \\ (https://creativecommons.org/licenses/by-nc/4.0/). See http://ivyspring.com/terms for full terms and conditions.
}

Received: 2018.08.02; Accepted: 2019.03.07; Published: 2019.05.12

\begin{abstract}
Objective: To evaluate the efficacy of induction chemotherapy in young adults with locally advanced oral squamous cell carcinoma (OSCC) and the usefulness of ERCCl as a prognostic indicator.

Methods: A total of 156 young adults with locally advanced OSCC were retrospectively analyzed from May 2007 to May 2017. Cisplatin based induction chemotherapy followed by surgery and upfront surgery were the primary treatment options for locally advanced OSCC. ERCCI was evaluated by immunohistochemistry. Multivariate analysis was performed to identify significant prognostic factors for the overall survival (OS) in young adults with locally advanced OSCC.

Results: Extracapsular spread (ECS) $(p<0.0001)$ and UICC staging $(p<0.0001)$ were critical prognostic factors for OS in young adults with locally advanced OSCC. The 5 -year OS was $83.2 \%$ in No patients received induction chemotherapy and $61.7 \%$ in N0 patients received upfront surgery $(p<0.05)$. Patients with a low ERCCl expression were more likely to benefit from induction chemotherapy, as the 5 -year OS was $22.4 \%$ in patients with a high ERCCl expression and $84.7 \%$ in patients with a low ERCCl expression, respectively $(p<0.0001)$. However, induction chemotherapy resulted in a higher 5 -year OS (84.7\%) than upfront surgery $(59.1 \%)$ in patients with a low ERCCI expression $(p=0.03)$.

Conclusions: Induction chemotherapy can improve the outcome of N0 patients. However, the ERCCl expression should be determined in young patients with locally advanced OSCC prior to induction chemotherapy, as it is a useful biomarker for predicting the outcome after induction chemotherapy.
\end{abstract}

Key words: young adult, oral cancer, ERCC1, induction chemotherapy

\section{Introduction}

The squamous carcinoma of the oral cavity is the most prevalent form of oral malignancies, accounting for $>90 \%$ of all oral mucosal malignancies. ${ }^{1}$ Currently, wide resection and radiotherapy have been considered the gold standards for the treatment of oral squamous cell carcinoma. However, chemotherapy is recommended only for high risk patients after surgery, and the 5-year overall survival (OS) has remained at about $55 \%$ over the past three decades. ${ }^{2,3}$ Recently, induction chemotherapy with 
docetaxel, cisplatin and fluorouracil has been shown to be useful for nonsurgical management of patients with head and neck squamous cell carcinoma (HNSCC). ${ }^{4}$ A randomized phase III trial of induction chemotherapy in locally advanced resectable oral squamous cell carcinoma (OSCC) has also demonstrated the favorable pathologic or clinical response to cisplatin-based chemotherapy. ${ }^{3}$

The optimal treatment options depend on many factors, including the size and location of the primary tumor, cervical lymph node (CLN) status, pathological differentiation, patient's tolerance to treatment and patient's desires., ${ }^{3,5}$ The prognostic value of age has rarely been studied as oral cancers occur most commonly in middle-aged and older patients, but seldom in young individuals. ${ }^{6}$ However, recent studies have reported an increased incidence of OSCC in young adults, especially in young white women. ${ }^{7-9}$ Young adults with OSCC typically have no risk factors like tobacco use, alcohol use, and human papillomavirus infection, which are likely to be clinically and histologically distinct from other OSCCs. ${ }^{10,11}$ However, the treatment and outcome of OSCC in young adults remain controversial. Some retrospective studies reported that the outcome and treatment option are similar in young and older OSCC patients,, 12 while some studies reported that young adults had an aggressive clinical phenotype and often required intensive multimodality treatments. ${ }^{13,14}$

In this study, we retrospectively analyzed the clinical features, treatments, and outcomes of 156 young adults with locally advanced (stage III or IVA, according to the Union for International Cancer Control (UICC) [2002]) OSCC from May 2007 to May 2017 in the 9th Hospital of Shanghai Jiaotong University, a leading center for the treatment of OSCC in China. Specifically, we aimed to (1) define the clinical features of OSCC in young adults; ${ }^{15}$ (2) evaluate the efficacy of induction chemotherapy in young adults with locally advanced OSCC, and (3) evaluate whether ERCC1 can be a prognostic indicator of OSCC in young adults.

\section{Patients and methods}

A total of 156 young adults with locally advanced (stage III or IVA) OSCC (87 males and 69 females aged $<40$ years at diagnosis) hospitalized in the 9th Hospital of Shanghai Jiaotong University from May 2007 to May 2017 were recruited for this study. The data collected included gender, age, primary site, tumor size, CLN status, therapy mode, ERCC1 expression and survival time. Patients were excluded if they were above 40 years of age, or had unresectable tumors or prior radiotherapy. The primary disease was diagnosed by biopsy. The CLN status was evaluated by clinical examination, ultrasound or radiological examination, including magnetic resonance imaging (MRI) and computed tomography. The final diagnosis was confirmed by the pathological examination after neck resection.

Of the 156 young patients, 89 received induction chemotherapy consisting of docetaxel $75 \mathrm{mg} / \mathrm{m}^{2}$ intravenously on day 1 , followed by cisplatin 75 $\mathrm{mg} / \mathrm{m}^{2}$ intravenously on day 1 and fluorouracil 750 $\mathrm{mg} / \mathrm{m}^{2} / \mathrm{d}$ as a 120 -h continuous intravenous infusion on days 1 through 5 . Induction chemotherapy was administered every 3 weeks for two cycles, followed by radical surgery and postoperative radiotherapy. The other 76 patients received only radical resection and radiotherapy. Radical resection of the primary lesion and full neck dissection (functional or radical) with appropriate reconstruction (pedicle or free flap) were performed (Fig. 1). The safety margins of the primary lesion were $1.5 \mathrm{~cm}$ away from the palpable margins. However, the safety margins were $1.0 \mathrm{~cm}$ away from the marks made before induction chemotherapy in patients who received induction chemotherapy (Fig. 2). Radiotherapy was initiated 4 to 6 weeks after surgery. Standard conformal or intensity-modulated radiotherapy was allowed at a dose of 1.8 to 2 Gy per day, 5 days per week, for 6 weeks (54 to 60Gy in total).

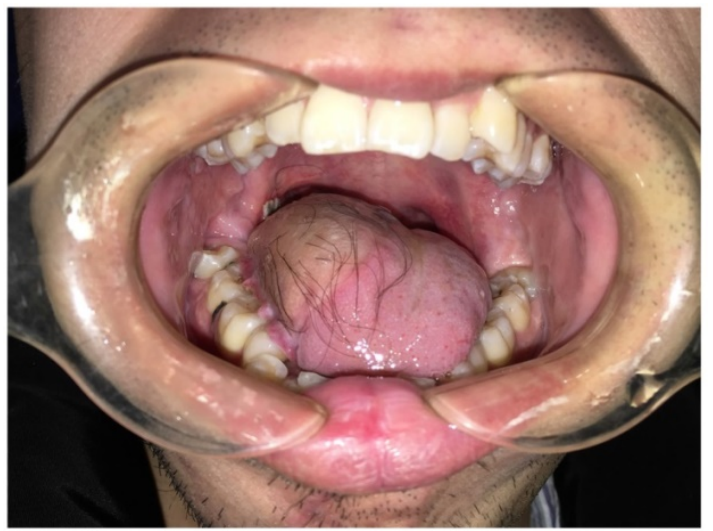

Figure 1: A forearm flap was performed for the reconstruction of tongue.

\section{ERCC1 expression}

The ERCC1 expression was evaluated by immunohistochemistry using the 8F1 monoclonal antibody (1: 80 dilutions, Neomarkers, USA). A semi-quantitative H-score (intensity * expression) was calculated for ERCC1 expression. To overcome variance in pre-analytic processing, the staining intensity of the nuclei of non-neoplastic basal epithelial cells (internal control, present in all samples) was designated $2+$ as previously described. The staining intensity of tumor cell nuclei was 
compared with the internal control on a scale of 0 to $3+$, with 0 representing no staining, and 3+ representing intense staining. The percentage of cells staining for ERCC1 was assigned a weighted expression score as described previously (None $=0$, $1-9 \%=1,10-49 \%=2$, and $50-100 \%=3$ ). The H-score $>=6$ indicated increased ERCC1 expression, while H-score $<6$ indicated decreased ERCC1 expression. ${ }^{16,17}$

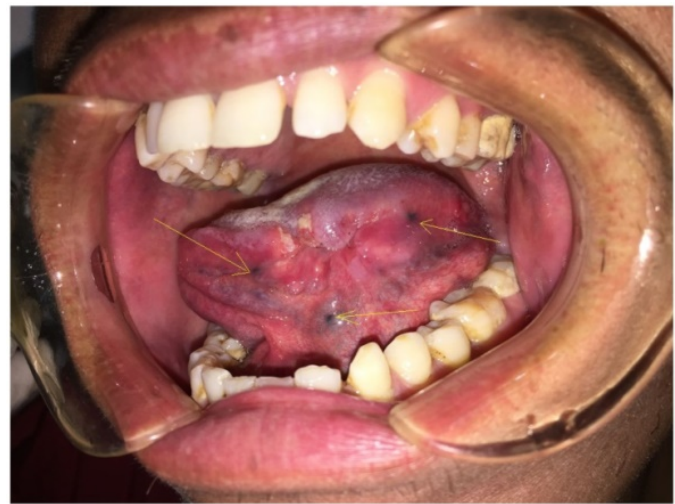

Figure 2: The marks was made $1.0 \mathrm{~cm}$ away from the tumor border before induction chemotherapy.

\section{Statistical analysis}

Survival was measured from the date of pathologic diagnosis, and patients still alive in May 2018 or lost in follow-up were censored. The outcome was defined by the overall survival (OS). Statistical analysis was performed using SAS 9.13. The survival rate was calculated by the Kaplan-Meier method. The prognostic variables considered in this study included gender, age, primary site, tumor size, CLN status, ERCC1 expression and therapy mode. The statistical significance of differences between survival curves was established by the log-rank test.

\section{Results}

\section{Patient characteristics}

The clinical and epidemiological characteristics of the 156 patients were shown in Table 1 . There were 87 males and 69 females with a male-to-female ratio of 1.26: 1 . The mean age at presentation was 33.2 years (range: 17-40 years); and the mean follow-up was 72 months (range: 12-132 months). Lesions occurred on tongue in 146 patients, gum in 6 patients and buccal in 4 patients, respectively. The 5 -year OS was $51.4 \%$ (Fig. $3)$, with no significant difference between genders, primary sites or T-stages. Heavy smoking was reported in only 11 male patients. Of the 18 patients received HPV testing, none showed a positive result. Three patients had a history of leukemia, and 2 patients had a history of nasopharyngeal carcinoma (NPC).
Table 1. 5-year OS by Prognostic Variables

\begin{tabular}{|c|c|c|c|c|}
\hline Variable & $\begin{array}{l}\text { NO. of } \\
\text { patient }\end{array}$ & 5-Year OS \% & $p$ & Log-rank \\
\hline Sex & & & 0.32 & - \\
\hline Men & 87 & 54.9 & & \\
\hline Women & 69 & 46.3 & & \\
\hline Primary site & & & 0.61 & - \\
\hline tongue & 146 & 47.2 & & \\
\hline gum & 6 & 49.4 & & \\
\hline buccal & 4 & 58.2 & & \\
\hline T-stage & & & 0.12 & - \\
\hline $\mathrm{T} 1$ or $\mathrm{T} 2$ & 68 & 62.2 & & \\
\hline $\mathrm{T} 3$ or $\mathrm{T} 4$ & 88 & 47.8 & & \\
\hline TNM-stage & & & $<0.0001$ & 24.49 \\
\hline stage III & 77 & 71.8 & & \\
\hline stage IVA & 79 & 31.8 & & \\
\hline CLN status & & & $<0.0001$ & 34.65 \\
\hline Positive with ECS & 18 & 8.9 & & \\
\hline $\begin{array}{l}\text { Positive without } \\
\text { ECS }\end{array}$ & 74 & 37.3 & & \\
\hline negative & 64 & 78.9 & & \\
\hline Induction chemo & & & 0.6 & - \\
\hline Yes & 89 & 52.5 & & \\
\hline No & 77 & 49.6 & & \\
\hline Chemo for N0 & & & 0.018 & 7.21 \\
\hline Yes & 39 & 83.2 & & \\
\hline No & 25 & 61.7 & & \\
\hline Chemo for $\mathrm{N}+$ & & & 0.09 & \\
\hline Yes & 50 & 16.7 & & \\
\hline No & 42 & 42.3 & & \\
\hline ERCC1 expression & & & $<0.0001$ & 29.94 \\
\hline Low & 48 & 84.7 & & \\
\hline High & 41 & 22.4 & & \\
\hline $\begin{array}{l}\text { Low ERCC1 } \\
\text { expression }\end{array}$ & & & 0.03 & 4.46 \\
\hline Chemo group & 41 & 84.7 & & \\
\hline $\begin{array}{l}\text { Upfront surgery } \\
\text { group }\end{array}$ & 24 & 59.1 & & \\
\hline
\end{tabular}

Abbreviations: CLN, cervical lymph nodes; N0, negative cervical lymph nodes; $\mathrm{N}+$, positive cervical lymph nodes; Chemo, chemotherapy.

\section{Extracapsular spread (ECS) in CLN was a poor prognostic factor}

The CLN status was evaluated by clinical examination, ultrasound or radiological examination, and then confirmed by neck dissection. Ten patients who were clinically N0 before treatment had positive CLN after neck dissection, while 8 patients who had clinically positive CLN before treatment had N0 diseases at the end. There were 64 patients with N0 diseases and 92 patients (58\%) with positive CLN. Of the 92 patients, ECS in CLN was found in 18 patients. These 18 patients showed the worst prognosis with a 5 -year OS of only 9\%; while the best prognosis was found in N0 patients, whose 5-year OS was as high as $79 \%$. The 5-year OS of patients with positive but without ECS was $37 \%$ (Log-Rank $=34.65, p<0.0001)$ (Fig. 4). Univariate analysis identified ECS in CLN as an independent poor prognostic factor for OS. Interestingly, of the 18 patients with ECS in CLN, 10 had a N1 stage and 8 had a N2 stage. 
UICC staging was a critical prognostic factor

According to the UICC-TNM staging system (version 2012), 77 patients were classified as stage III with a 5-year OS of $72 \%$, while 79 patients were classified as stage IVA with a 5-year OS of 32\% (Log-rank=24.49, $p<0.0001$ ) (Fig. 5). Univariate analysis showed that UICC staging was an independent prognostic factor for OS.

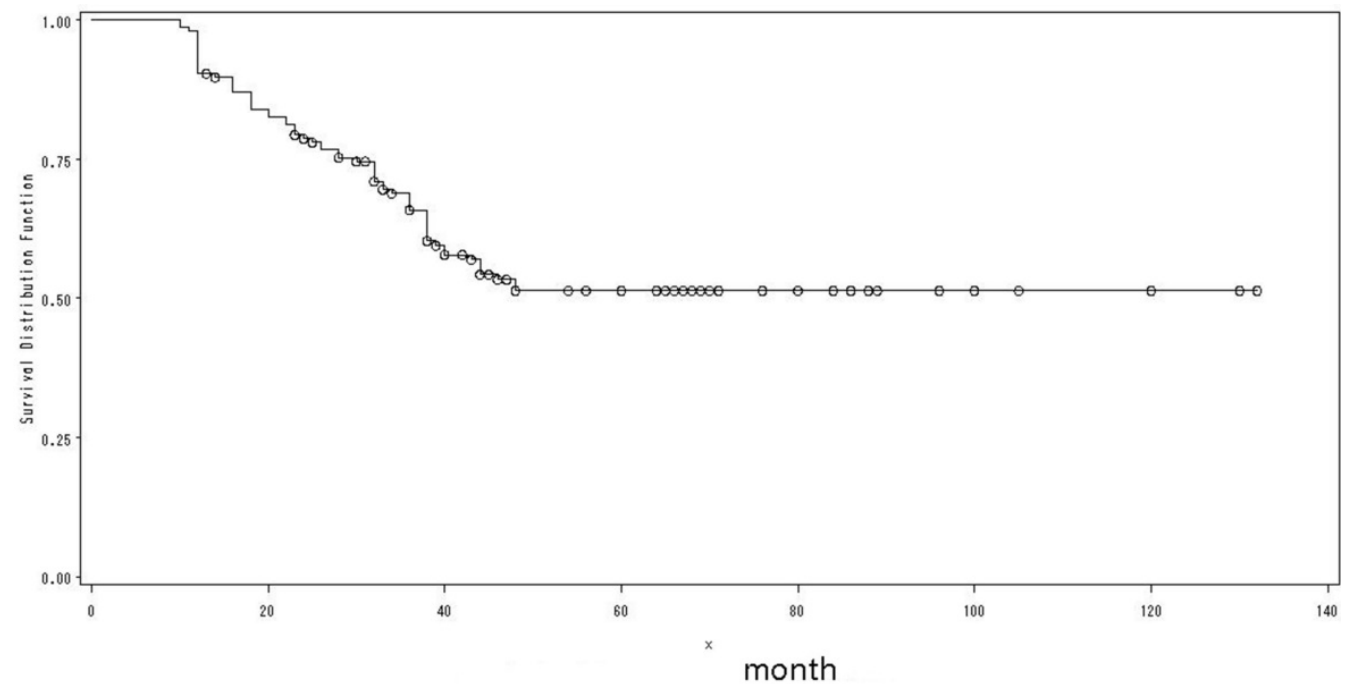

Figure 3: The overall survival of all patients.

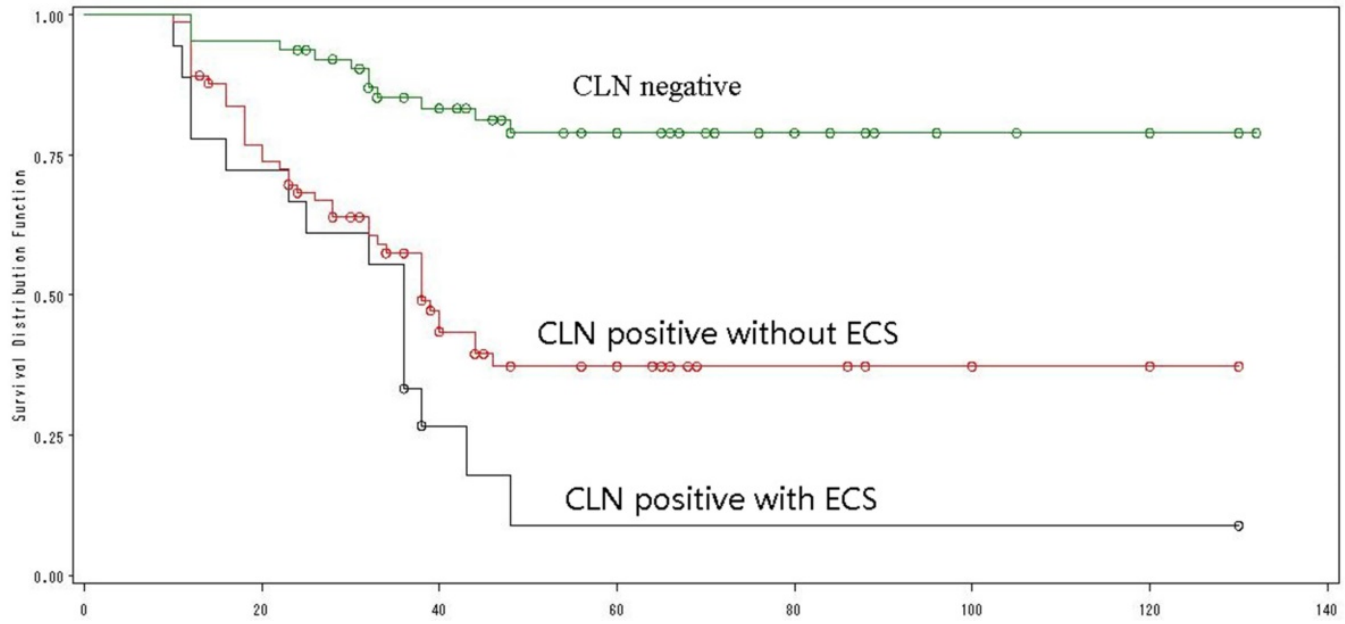

Figure 4: The overall survival by CLN status.

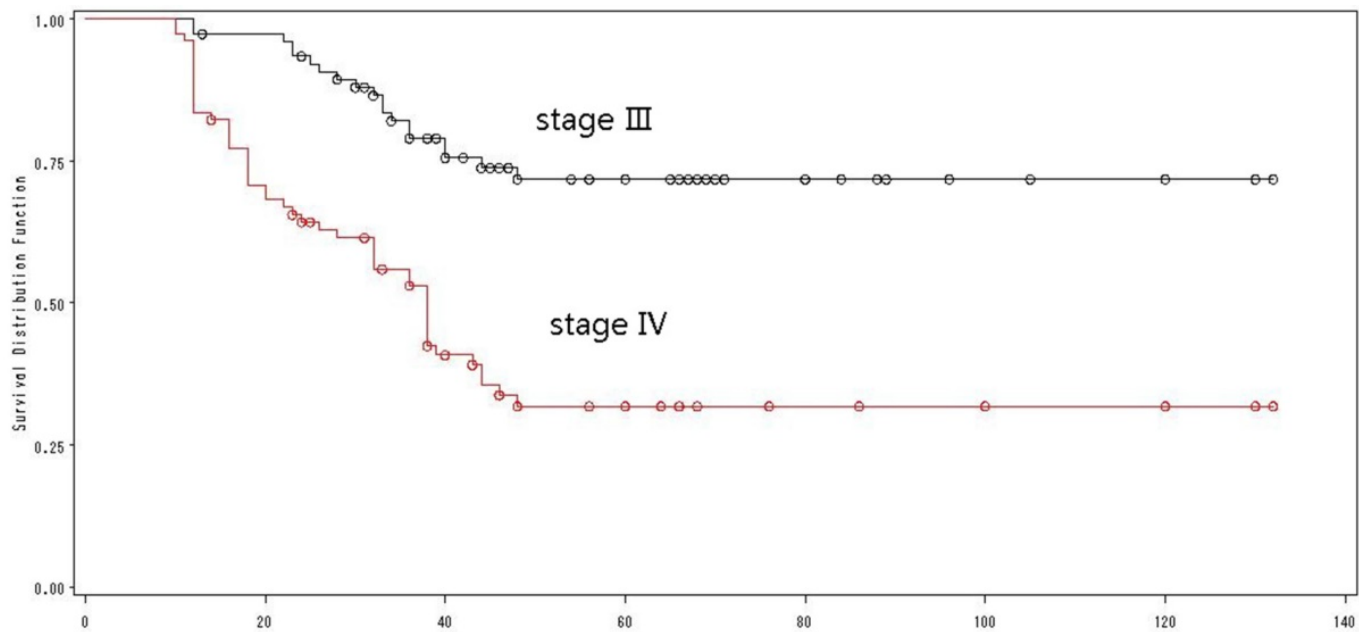

Figure 5: The overall survival by TNM-staging. 


\section{Induction chemotherapy couldn't improve the outcome of patients}

A total of 89 patients received induction chemotherapy, and the response was in accordance with RSICIST 1.1. However, there was no significant difference in the 5-year OS between patients with and without induction chemotherapy $(p=0.60)$, indicating that induction chemotherapy could not improve the outcome in young adults with locally advanced OSCC.

\section{Induction chemotherapy for N0 patients}

Of the 64 patients with N0 OSCCs, 39 received induction chemotherapy with a 5 -year OS of $83.2 \%$, while the rest 25 patients without induction chemotherapy had a 5-year OS of $61.7 \%$ (Log-rank=7.21, $p=0.018$ ) (Fig. 6), indicating that induction chemotherapy could improve the OS in young adults with NO OSCCs.

\section{Induction chemotherapy for CLN positive patients}

Fifty out of 92 patients with positive CLN received induction chemotherapy, and there was no significant difference in the 5-year OS between patients with and without induction chemotherapy $(16.7 \%$ versus $42.3 \%, p=0.09)$.

\section{Patients with a lower ERCCI expression were more likely to benefit from induction chemotherapy}

Of the 138 patients received ERCC1 testing, 73 had a high ERCC1 expression (Fig 7), and 65 had a low ERCC1 expression (Fig 8). Of the 89 patients received induction chemotherapy, 48 patients had a high ERCC1 expression, and 41 had a low ERCC1 expression. The 5 -year OS was $22.4 \%$ in patients with a high ERCC1 expression and $84.7 \%$ in patients with a low ERCC1 expression (Log-rank=29.94, $p<0.0001$ ) (Fig. 9), indicating that patients with a lower ERCC1 expression were more likely to benefit from induction chemotherapy.

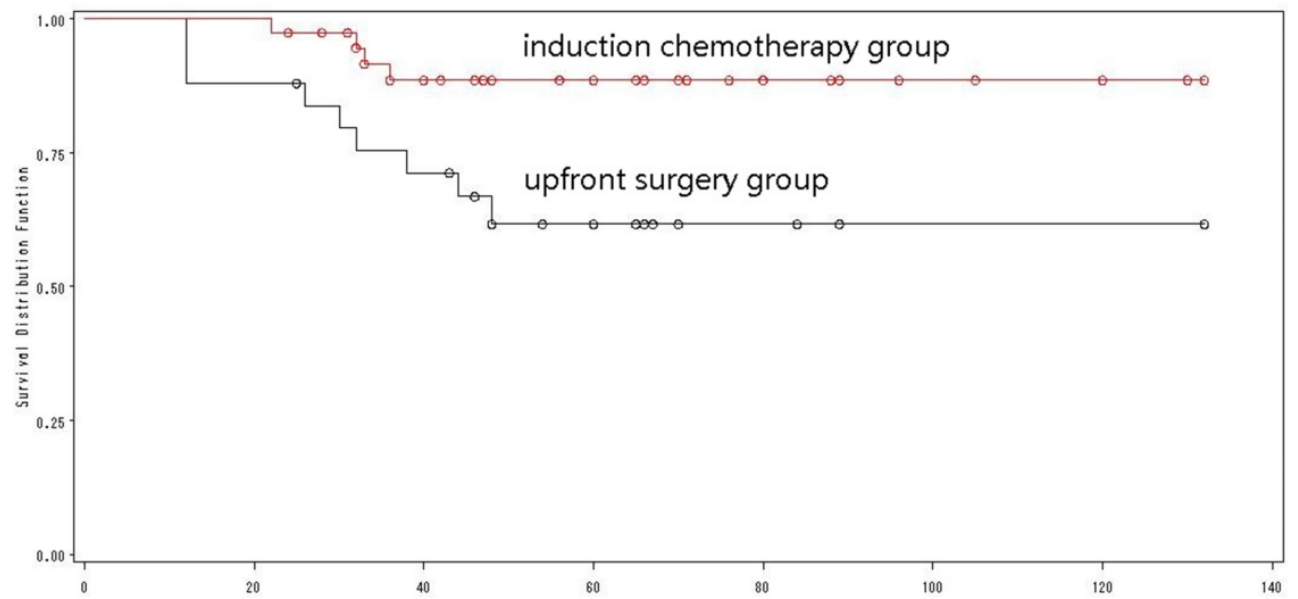

Figure 6: The overall survival of N0 patients by treatment mode.

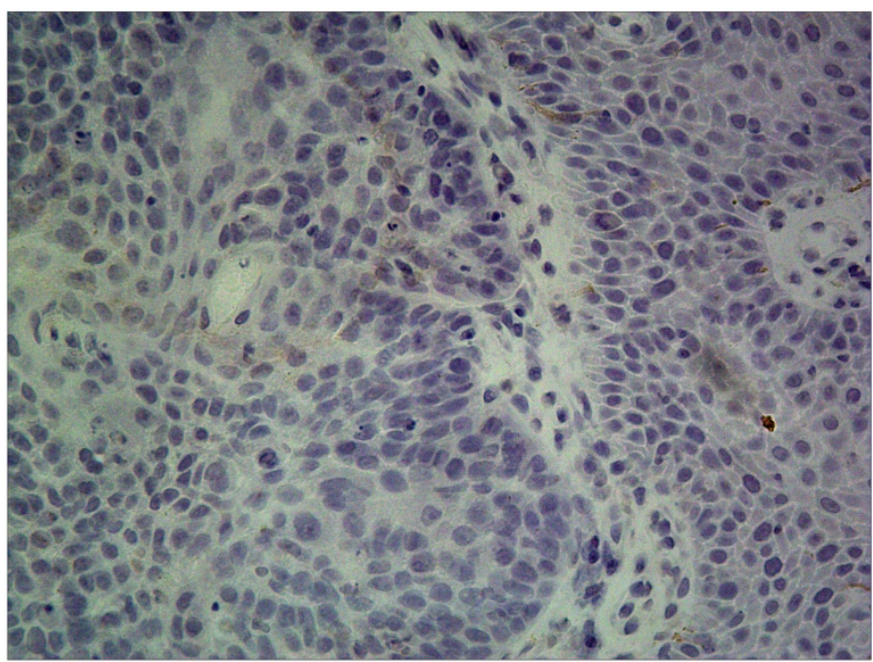

Figure 7: High ERCCl expression: the percentage of cells staining for $E R C C l$ was 2 points, with intense staining $(3+)$,and the $\mathrm{H}$-score $>=6$. 


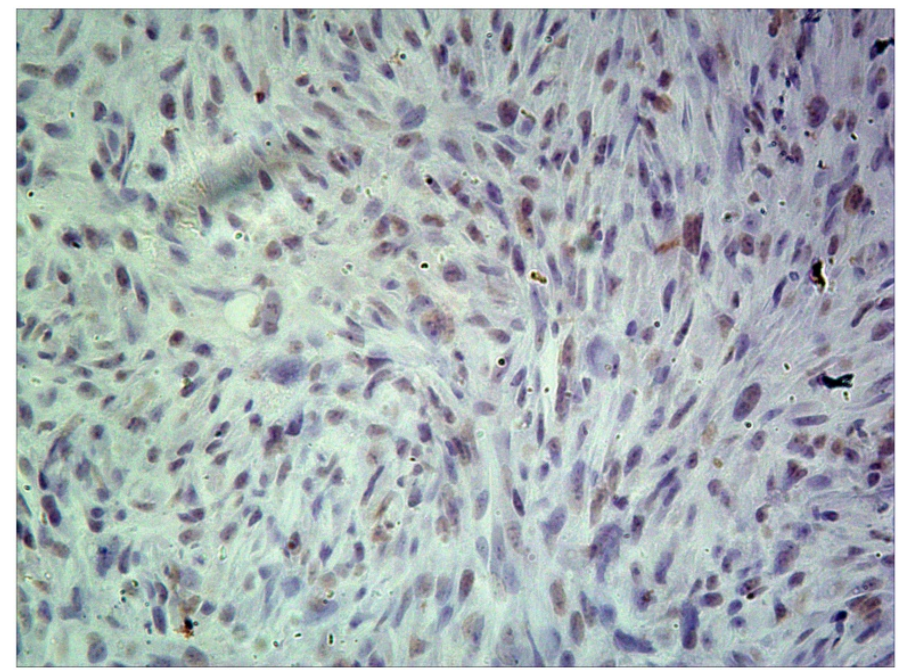

Figure 8: Low ERCCl expression: the percentage of cells staining for $E R C C l$ was 2 points, with no staining, and the $\mathrm{H}$-score $=0$.

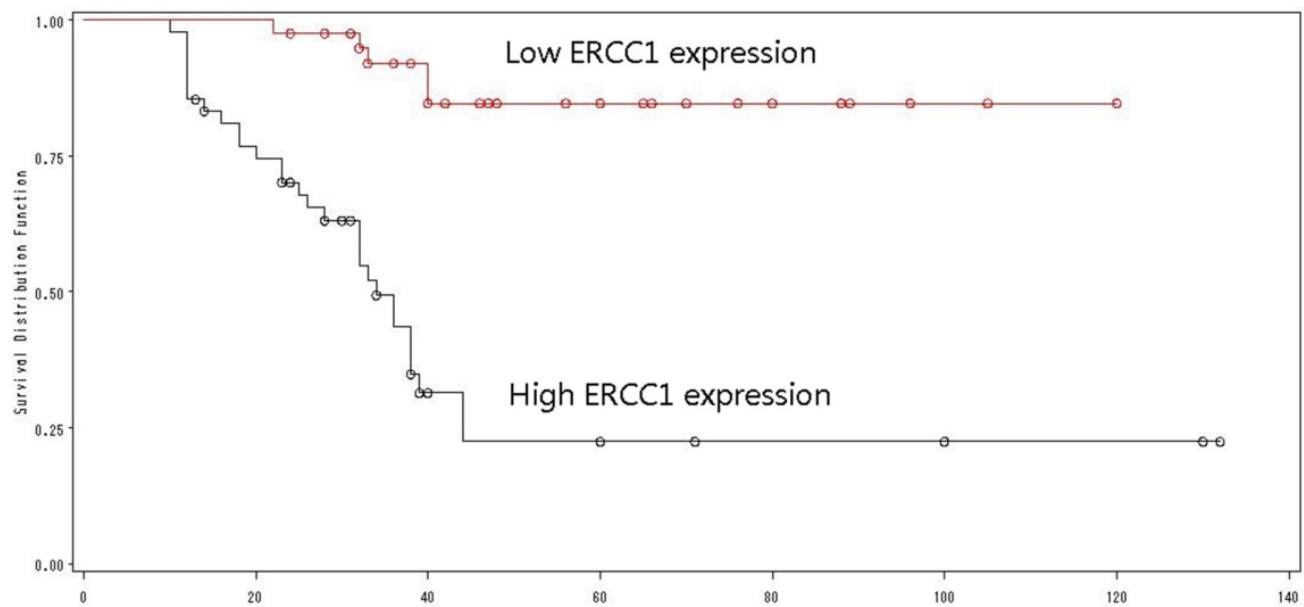

Figure 9: The overall survival by ERCCl expression.

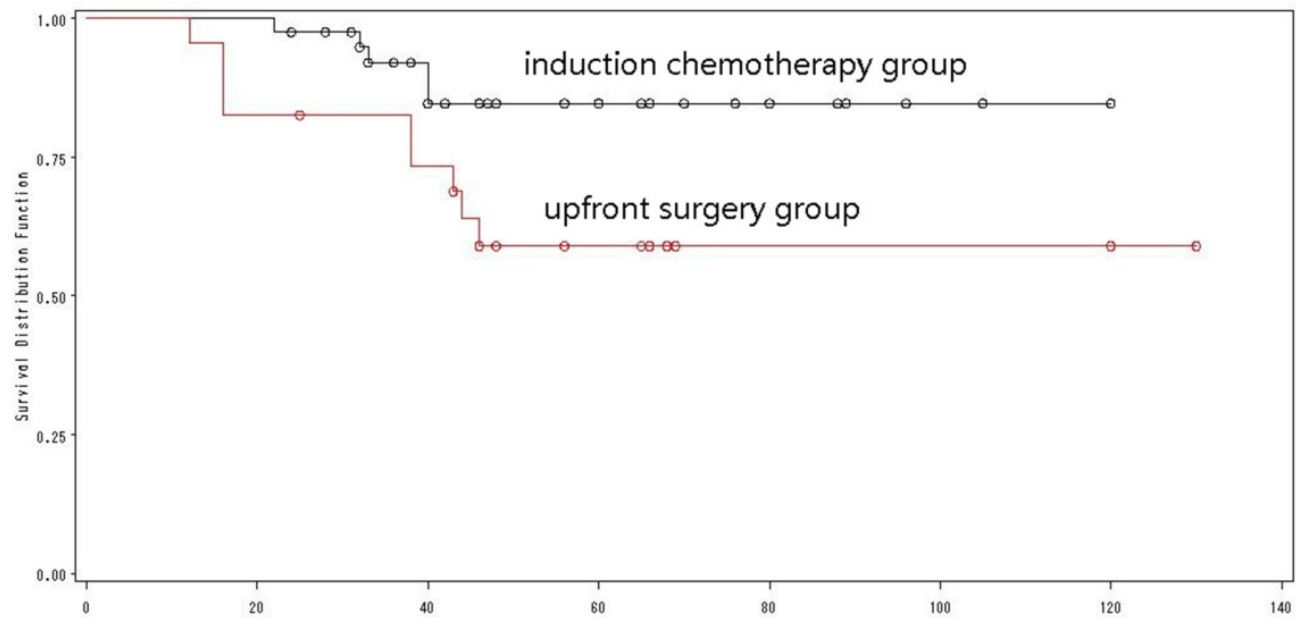

Figure 10: The overall survival of low ERCCl expressions by treatment mode.

Induction chemotherapy modified by ERCC1 improved the outcome

Of the 65 patients with a low ERCC1 expression, 41 received induction chemotherapy, and 24 did not
(Table 2). There was a significant difference in the 5 -year OS between the two groups $(84.7 \%$ versus $59.1 \%$, Log-rank=4.46, $p=0.03$ ) (Fig. 10). Multivariate analysis with the Cox proportional hazard model was used to validate the outcome, and 
demonstrated that induction chemotherapy was an independent prognostic factor that improving the outcome of young adults with low expression of ERCC1.

Table 2. 5-Year OS of low ERCCl expression by prognostic variables.

\begin{tabular}{llll}
\hline & $\begin{array}{l}\text { NO. of } \\
\text { patients }\end{array}$ & Un, chi-square & \\
\cline { 3 - 4 } Variable & & $0.06,3.40$ & $0.782,1.62$ \\
T1 or T2 & 35 & & \\
T3 or T4 & 30 & & $0.0001,14.94$ \\
TNM-stage & & $0.02,8.15$ & \\
stage III & 39 & & $<0.0001,17.16$ \\
stage IVA & 26 & & \\
CLN status & & $<0.0001,17.41$ & \\
Positive & 25 & & $0.0297,4.73$ \\
negative & 40 & $0.03,6.17$ & \\
Low ERCC1 expression & & & \\
Chemo group & 41 & & \\
Upfront surgery group & 24 & & \\
\hline
\end{tabular}

\section{Treatment failure and AEs}

Nine patients $(6 \%)$ were lost to follow up. Myelosuppression occurred in 28 patients (31.4\%), while liver injury occurred in 12 patients (13.4\%). However, no irreversible myelosuppression and liver injury were found. Totally 68 patients $(44 \%)$ died, 65 of whom died of local recurrence, and 3 of whom died of distant metastases.

\section{Discussion}

OSCC occurs predominantly in middle-aged and older individuals with a long history of tobacco or alcohol use.6,18,19 However, recent studies have reported an increased incidence of OSCC in young adults. $^{7-9}$ Tobacco or alcohol use may not be the etiology of OSCC in young adults, as our study has demonstrated that only $7 \%(11 / 156)$ patients had heavy smoking. HPV infection, which has been thought to be a potential factor of OSCC in middle-aged and older patients, was not found in young adults with locally advanced OSCC in this study. Other possible causal factors include genetic abnormalities, oncogenic viral infections, and/or other environmental exposures. ${ }^{9}$ In our study, five patients suffered from other malignant cancers such as leukemia and NPC before the diagnosis of OSCC, which may be associated with the immunodeficiency.

There is a male predominance in the incidence of OSCC, with a male-to-female ratio of about 2: 1. 3,20,21 However, the incidence of OSCC has been increasing in young white women, with a male-to-female ratio of 1.6: $1.9^{9}$ In this study, the male-to-female ratio is $1.3: 1$. Despite the high incidence of OSCC in young women, gender is not a significant prognostic factor in this study. However, previous studies have suggested that gender can significantly predict occult nodal, and women are more likely to have occult nodal diseases. ${ }^{22}$

In this study, we found that 10 patients who were clinically N0 before treatment had positive CLN after neck dissection. The CLN status is thought to be the most accurate predictor of OS in OSCC patients. ${ }^{23}$ Especially, the outcome is worse when ECS is present in the positive CLN. ${ }^{24,25}$ This is also supported by our results. Pinsolle et al. reported a strong relationship between clinical $\mathrm{N}$ classification and ECS, and ECS was present in $35 \%$ of clinical N1 patients, $55 \%$ of clinical N2 patients and 74\% of clinical N3 patients, respectively. ${ }^{26}$ However, the association between clinical $\mathrm{N}$ classification and ECS is not confirmed in our study.

Induction chemotherapy could not improve the OS of all patients with locally advanced OSCC, but it can improve the outcome of patients with a good pathological or clinical response to chemotherapy. ${ }^{3}$ In this study, induction chemotherapy also resulted in no improvement in OS of all young patients with locally advanced OSCC, but it improved the OS of N0 patients. N0 is confirmed by neck dissection rather than the primary clinical $\mathrm{N}$ classification in this study. Meanwhile, the primary clinical $\mathrm{N}$ classification has an error with the real node status confirmed by neck dissection. Therefore, both favorable pathological or clinical response to chemotherapy and real nodes status are the results after treatment. Thus, it is critically important to identify a useful predictor biomarker for induction chemotherapy.

Cisplatin is the most commonly used chemotherapy for locally advanced OSCC. Cisplatin resistance is one of the most important factors for the failure of induction chemotherapy, which can be caused by increased DNA repair. ${ }^{27-30}$ ERCC1 is the first human DNA repair gene identified by molecular cloning, and ERCC1 expression is negatively correlated with the survival and/or responsiveness to cisplatin-based regimens in several human neoplasms including bladder, colorectal, gastric, head and neck, and non-small cell lung cancers. ${ }^{31-34}$ In our study, we have also found that patients with a lower ERCC1 expression are more likely to benefit from cisplatin-based induction chemotherapy. In patients with a low ERCC1 expression, induction chemotherapy resulted in a better outcome than upfront surgery. Therefore, ERCC1 expression can be a useful predictor biomarker for induction chemotherapy.

In conclusion, induction chemotherapy could not improve the outcome of all young adults with locally advanced OSCC. However, it would be a good 
choice for them to have an ERCC1 test before treatment, as the ERCC1 expression is a useful biomarker for predicting the outcome after induction chemotherapy.

The study was a retrospective study, and the findings of induction chemotherapy in young adults with locally advanced OSCC and ERCC1 as a prognostic indicator must be validated in our future prospective studies.

\section{Acknowledgements}

This work was supported by Shanghai Health and Family Planning Commission [No.20154Y0057].

\section{Competing Interests}

The authors have declared that no competing interest exists.

\section{References}

1. Bradley PJ: Primary malignant mucosal melanoma of the head and neck. Curr Opin Otolaryngol Head Neck Surg.2006; 14:100-4.

2. Parkin DM, Bray F, Ferlay J, et al: Global cancer statistics, 2002. CA Cancer J Clin.2005; 55:74-108.

3. Zhong LP, Zhang CP, Ren GX, et al: Randomized phase III trial of induction chemotherapy with docetaxel, cisplatin, and fluorouracil followed by surgery versus up-front surgery in locally advanced resectable oral squamous cell carcinoma. J Clin Oncol.2013; 31:744-51.

4. Lorch $\mathrm{JH}$, Goloubeva $\mathrm{O}$, Haddad RI, et al: Induction chemotherapy with cisplatin and fluorouracil alone or in combination with docetaxel in locally advanced squamous-cell cancer of the head and neck: long-term results of the TAX 324 randomised phase 3 trial. Lancet Oncol.2011; 12:153-9.

5. Neville BW, Day TA: Oral cancer and precancerous lesions. CA Cancer J Clin.2002;52:195-215

6. Chen JK, Katz RV, Krutchkoff DJ: Intraoral squamous cell carcinoma. Epidemiologic patterns in Connecticut from 1935 to 1985. Cancer.1990; 66:1288-96.

7. Shiboski CH, Schmidt BL, Jordan RC: Tongue and tonsil carcinoma: increasing trends in the U.S. population ages 20-44 years. Cancer.2005; 103:1843-9.

8. Moller H: Changing incidence of cancer of the tongue, oral cavity, and pharynx in Denmark. J Oral Pathol Med .1989;18:224-9.

9. Patel SC, Carpenter WR, Tyree S, et al: Increasing incidence of oral tongue squamous cell carcinoma in young white women, age 18 to 44 years. J Clin Oncol. 2011;29:1488-94.

10. Bragelmann J, Dagogo-Jack I, El Dinali M, et al: Oral cavity tumors in younger patients show a poor prognosis and do not contain viral RNA. Oral Oncol. 2013; 49:525-33.

11. Rautava J, Luukkaa M, Heikinheimo K, et al: Squamous cell carcinomas arising from different types of oral epithelia differ in their tumor and patient characteristics and survival. Oral Oncol. 2007;43:911-9.

12. Garavello W, Spreafico R, Gaini RM: Oral tongue cancer in young patients: a matched analysis. Oral Oncol. 2007; 43:894-7.

13. Salem A: Dismissing links between HPV and aggressive tongue cancer in young patients. Ann Oncol. 2010; 21:13-7.

14. Chitapanarux I, Lorvidhaya V, Sittitrai $P$, et al: Oral cavity cancers at a young age: analysis of patient, tumor and treatment characteristics in Chiang Mai University Hospital. Oral Oncol. 2006; 42:83-8.

15. Joshi MB, Shirota Y, Danenberg KD, et al: High gene expression of TS1, GSTP1, and ERCC1 are risk factors for survival in patients treated with trimodality therapy for esophageal cancer. Clin Cancer Res. 2005; 11:2215-21.

16. Handra-Luca A, Hernandez J, Mountzios G, et al: Excision repair cross complementation group 1 immunohistochemical expression predicts objective response and cancer-specific survival in patients treated by Cisplatin-based induction chemotherapy for locally advanced head and neck squamous cell carcinoma. Clin Cancer Res. 2007; 13:3855-9.

17. Hao D, Lau HY, Eliasziw M, et al: Comparing ERCC1 protein expression, mRNA levels, and genotype in squamous cell carcinomas of the head and neck treated with concurrent chemoradiation stratified by HPV status. Head Neck. 2012:34:785-91.

18. Mashberg A, Boffetta P, Winkelman R, et al: Tobacco smoking, alcohol drinking, and cancer of the oral cavity and oropharynx among U.S. veterans. Cancer. 1993; 72:1369-75.

19. Beynon RA, Lang S, Schimansky S, et al: Tobacco smoking and alcohol drinking at diagnosis of head and neck cancer and all-cause mortality: Results from head and neck 5000, a prospective observational cohort of people with head and neck cancer. Int J Cancer. 2018; Accepted Article.

20. Licitra L, Grandi C, Guzzo M, et al: Primary chemotherapy in resectable oral cavity squamous cell cancer: a randomized controlled trial. J Clin Oncol.2003; 21:327-33.

21. Lee DY, Kang SH, Kim JH, et al: Survival and recurrence of resectable tongue cancer: Resection margin cutoff value by T classification. Head Neck.2018; 40:283-291.

22. Zhan KY, Morgan PF, Neskey DM, et al: Preoperative predictors of occult nodal disease in cT1N0 oral cavity squamous cell carcinoma: Review of 2623 cases. Head Neck. 2018; 40: 1967-76.

23. Marur S, Forastiere AA: Head and Neck Squamous Cell Carcinoma: Update on Epidemiology, Diagnosis, and Treatment. Mayo Clin Proc. 2016; 91:386-96.

24. Ferlito A, Rinaldo A, Devaney KO, et al: Prognostic significance of microscopic and macroscopic extracapsular spread from metastatic tumor in the cervical lymph nodes. Oral Oncol. 2002; 38:747-51.

25. Puri SK, Fan CY, Hanna E: Significance of extracapsular lymph node metastases in patients with head and neck squamous cell carcinoma. Curr Opin Otolaryngol Head Neck Surg. 2003; 11:119-23.

26. Pinsolle J, Pinsolle V, Majoufre C, et al: Prognostic value of histologic findings in neck dissections for squamous cell carcinoma. Arch Otolaryngol Head Neck Surg. 1997; 123:145-8.

27. Amable L: Cisplatin resistance and opportunities for precision medicine. Pharmacol Res,2016; 106:27-36.

28. de Graeff A, Slebos RJ, Rodenhuis S: Resistance to cisplatin and analogues: mechanisms and potential clinical implications. Cancer Chemother Pharmacol.1988; 22:325-32.

29. Ferry KV, Hamilton TC, Johnson SW: Increased nucleotide excision repair in cisplatin-resistant ovarian cancer cells: role of ERCC1-XPF. Biochem Pharmacol. 2000; 60:1305-13.

30. Kelland LR, Mistry P, Abel G, et al: Establishment and characterization of an in vitro model of acquired resistance to cisplatin in a human testicular nonseminomatous germ cell line. Cancer Res.1992; 52:1710-6.

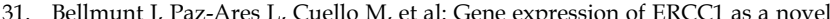
prognostic marker in advanced bladder cancer patients receiving cisplatin-based chemotherapy. Ann Oncol.2007;18:522-8.

32. Shirota Y, Stoehlmacher J, Brabender J, et al: ERCC1 and thymidylate synthase mRNA levels predict survival for colorectal cancer patients receiving combination oxaliplatin and fluorouracil chemotherapy. J Clin Oncol. 2001; 19:4298-304

33. Metzger R, Leichman CG, Danenberg KD, et al: ERCC1 mRNA levels complement thymidylate synthase mRNA levels in predicting response and survival for gastric cancer patients receiving combination cisplatin and fluorouracil chemotherapy. J Clin Oncol.1998;16:309-16.

34. Olaussen KA, Dunant A, Fouret P, et al: DNA repair by ERCC1 in non-small-cell lung cancer and cisplatin-based adjuvant chemotherapy. $\mathrm{N}$ Engl J Med. 2006;355:983-91. 